\title{
Corrigendum: Protein and Organic-Molecular Crystallography with 300kV Electrons on a Direct Electron Detector
}

\author{
Kiyofumi Takaba ${ }^{1}$, Saori Maki-Yonekura ${ }^{1}$, Satoru Inoue ${ }^{2}$, Tatsuo Hasegawa ${ }^{2}$ and \\ Koji Yonekura ${ }^{1,3,4 *}$
}

${ }^{1}$ Biostructural Mechanism Laboratory, RIKEN SPring-8 Center, Sayo, Japan, ${ }^{2}$ Department of Applied Physics, The University of Tokyo, Tokyo, Japan, ${ }^{3}$ Advanced Electron Microscope Development Unit, RIKEN-JEOL Collaboration Center, RIKEN Baton Zone Program, Sayo, Japan, ${ }^{4}$ Institute of Multidisciplinary Research for Advanced Materials, Tohoku University, Sendai, Japan

Keywords: DE64, energy filter, electron 3D crystallography (3D ED/MicroED), eEFD, CRYO ARM

\section{Corrigendum on}

Protein and Organic-Molecular Crystallography With $300 \mathrm{kV}$ Electrons on a Direct Electron Detector

by Takaba, K., Maki-Yonekura, S., Inoue, S., Hasegawa, T., and, Yonekura, K (2021). Front. Mol. Biosci. 7:612226. doi: 10.3389/fmolb.2020.612226

\section{OPEN ACCESS}

Edited and reviewed by: Dan Shi,

Center for Cancer Research, United States

${ }^{*}$ Correspondence: Koji Yonekura yone@spring8.or.jp

Specialty section: This article was submitted to

Structural Biology,

a section of the journal Frontiers in Molecular Biosciences

Received: 29 July 2021 Accepted: 05 August 2021 Published: 18 August 2021

Citation: Takaba K, Maki-Yonekura S, Inoue S, Hasegawa T and Yonekura K (2021)

Corrigendum: Protein and OrganicMolecular Crystallography with $300 \mathrm{kV}$

Electrons on a Direct Electron Detector.

Front. Mol. Biosci. 8:749448. doi: 10.3389/fmolb.2021.749448
In the original article, there was an error in the Summary and Perspectives. It was indicated that radiation damage caused by single $300 \mathrm{kV}$ electron is reduced to 96 and 25\%, compared with 100 and $200 \mathrm{kV}$, respectively. In fact, the correct values are by 49 and $20 \%$. A correction has been made to the Summary and Perspectives section, paragraph 2.

"Radiation damage is serious in both X-ray analysis and cryo-EM, and previous studies observed that even a small amount of electron irradiation caused breaks of cysteine bonds (Hattne et al., 2018, 2019) and reduction of metal (Yonekura et al., 2015) in protein crystals. The radiation damage caused by single $300 \mathrm{kV}$ electron is reduced by 49 and 20\%, compared with 100 and $200 \mathrm{kV}$, respectively (Yonekura et al., 2019). Deposited energy with single $300 \mathrm{kV}$ electron $/ \AA^{2}$ was calculated to $5.6 \times 10^{6} \mathrm{~Gy}(\mathrm{~J} / \mathrm{kg})$ for water (ICRU, 2014; Yonekura et al., 2019), where Gy is a standard unit in X-ray crystallography and related areas. Henderson limit, a criterion for a tolerable energy deposition on biological samples and widely used in X-ray crystallography, is $\sim 2 \times 10^{7} \mathrm{~Gy}$ (Henderson, 1990). The catalase structure here was obtained from a maximum exposure of $3.5 \times 10^{6} \mathrm{M}$ Gy for single dataset (Table 1), and this is $1 / 5.7$ of Hendrson limit. Thus, our system would be suitable for electron 3D crystallography with less damaging, a smaller point spread, and less noise than using the scintillator coupled camera."

The authors apologize for this error and state that this does not change the scientific conclusions of the article in any way. The original article has been updated.

Publisher's Note: All claims expressed in this article are solely those of the authors and do not necessarily represent those of their affiliated organizations, or those of the publisher, the editors and the reviewers. Any product that may be evaluated in this article, or claim that may be made by its manufacturer, is not guaranteed or endorsed by the publisher.

Copyright (๑) 2021 Takaba, Maki-Yonekura, Inoue, Hasegawa and Yonekura. This is an open-access article distributed under the terms of the Creative Commons Attribution License (CC BY). The use, distribution or reproduction in other forums is permitted, provided the original author(s) and the copyright owner(s) are credited and that the original publication in this journal is cited, in accordance with accepted academic practice. No use, distribution or reproduction is permitted which does not comply with these terms. 